\title{
Carbon dots have antitumor action as monotherapy or combination therapy
}

\author{
Jufeng Xia ${ }^{1,2, *}$, Yasuyuki Kawamura ${ }^{2}$, Tomoaki Suehiro ${ }^{2}$, Yu Chen ${ }^{1}$, Kunimichi Sato ${ }^{2}$ \\ ' Graduate School of Frontier Science, The University of Tokyo, Chiba, Japan; \\ ${ }^{2}$ New Japan Medical Institute, Bunkyo City, Tokyo, Japan.
}

\begin{abstract}
Summary Carbon dots prepared from different sources have been widely studied in various medical applications. Those dots have been reported to be able to inhibit the proliferation of cancer cells, such as prostate cancer cells. The current study used carbon dots prepared from red beans to determine their effect on 16 cell lines including liver cancer cells, pancreatic cancer cells, intrahepatic cholangiocarcinoma cells, and colorectal adenocarcinoma cells. In a cellular viability experiment, carbon dots were found suppress cancer cell viability in a time- and dose-dependent manner. In a cell migration experiment, cancer cells treated with carbon dots had less ability to heal, suggesting that carbon dots inhibit cell migration. In another cellular viability experiment, a combination of carbon dots and doxorubicin resulted in greater inhibition than cells treated with either therapy alone. These findings suggest that carbon dots could be an alternative and complementary medicine for the treatment of cancers.
\end{abstract}

Keywords: Carbon dots, liver cancer, pancreatic cancer, intrahepatic cholangiocarcinoma, colorectal adenocarcinoma, combination therapy

\section{Introduction}

Cancer is a group of diseases involving abnormal cell growth with the potential to invade or spread to other parts of the body (1). In 2018, 18.1 million new cases of cancer and 9.6 million deaths occurred globally. About $20 \%$ of males and $17 \%$ of females will develop cancer at some point in time, while $13 \%$ of males and $9 \%$ of females will die from it (2). There are many options for cancer treatment; the primary ones include surgery, chemotherapy, radiation therapy, and targeted therapy (3-7). However, current therapeutic methods have several drawbacks, such as the inability to kill microscopic disease, damage to nearby normal tissues, toxicities and adverse reactions, and resistance. Based on the limitations of those options, increasing attention is being devoted to the development of novel drugs and medical materials (8).

\footnotetext{
*Address correspondence to:

Dr. Jufeng Xia, Department of Human and Engineered Environmental Studies, Graduate School of Frontier Sciences, The University of Tokyo, 5-1-5 Kashiwa-no-ha, Kashiwa Chiba 227-8568, Japan.

E-mail: xia-tky@umin.ac.jp
}

Due to their unique physicochemical features and their ability to enter cells, carbon nanomaterials possess great potential for biomedical applications (9-11). The utilization of carbon nanomaterials as innovative drug delivery systems for chemotherapeutics and other bioactive compounds has extensively been studied over the past few years (12-14). Studies have suggested that carbon-based materials, such as carbon fibers, carbon nanotubes, and carbon dots, are able to enhance apoptosis and inhibit proliferation of cancer cells through a series of signaling pathways $(15,16)$.

Carbon dots prepared from different sources have been widely studied in imaging and drug delivery but less so in terms of intracellular activity and mechanisms of potential anticancer action (17). As an example, carbon dots prepared from a spice have been found to be able to inhibit tumor growth in a xenograft model (17). Another study found that carbon dots prepared from tea can bind to certain amino acids on ARF and colocalize with ARF in the nucleus, thus sensitizing cancer cells to rapamycin $(18,19)$. The current study used an atomic carbon material (Australian patent number: 2005230336, Figure 1). Carbon dots were prepared from red beans to determine if they could inhibit the proliferation and migration of several cancer 


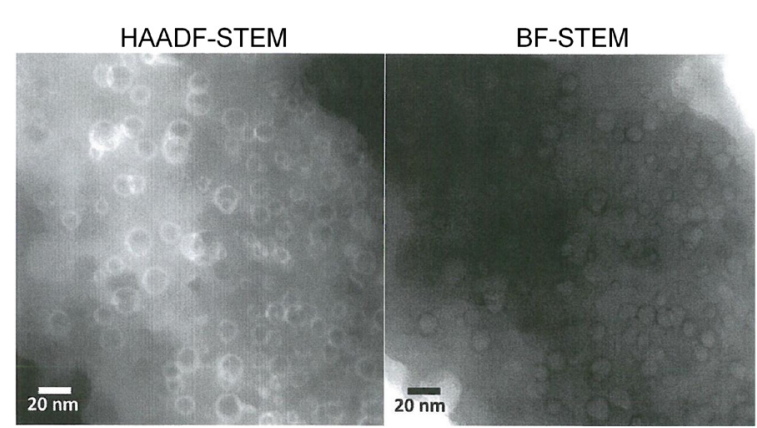

Figure 1. STEM model. HAADF-STEM: High-angle annular dark-field scanning transmission electron microscopy. BFSTEM: Bright-field scanning transmission electron microscopy.

cell lines and if they could promote the inhibitory effect of a conventional chemotherapy drug when administered in combination.

\section{Materials and Methods}

\subsection{Reagents}

High-glucose Dulbecco's modified Eagle's medium (DMEM) and fetal bovine serum (FBS) were purchased from Gibco (Gaithersburg, MD, USA). Penicillin and streptomycin were also purchased from Gibco (Gaithersburg, MD, USA). Cell Proliferation Kit I was purchased from Roche Applied Science (Penzberg, Upper Bavaria, Germany). Doxorubicin was purchased from Sigma (St. Louis, MO, USA). Carbon dots were donated by the New Japan Medical Institute (Bunkyo, Tokyo, Japan).

\subsection{Cell lines and maintenance}

Liver cancer cell lines (HepG2, HepG2.2.15, PLC/ PRF/5, Huh-7, Huh-1, HLE, HLF, SK-Hep-1, Hep3B, and BEL-7402), pancreatic cancer cell lines (Panc-1, CAPAN-1, MIA-Paca-2, and SUIT-2), an intrahepatic cholangiocarcinoma cell line (RBE), and a colorectal adenocarcinoma cell line (DLD-1) were used in this study. These cell lines were maintained in DMEM medium supplemented with $10 \%$ fetal bovine serum, $100 \mathrm{unit} / \mathrm{mL}$ penicillin, and $100 \mathrm{mg} / \mathrm{mL}$ streptomycin at $37^{\circ} \mathrm{C}$ in a humidified incubator with a $5 \% \mathrm{CO}_{2}$ atmosphere.

\subsection{Dispersion of carbon dots}

Dispersions of carbon dots were prepared using human serum albumin (HSA; Baxter, Unterschleissheim, Germany). HSA was diluted with phosphate-buffered saline (PBS) and added to the carbon dots at a ratio of 1:1 by weight, followed by sonication with an ultrasonic tip (Sonoplus HD 2070, Bandelin; Heinrichstra, Berlin, Germany) for $5 \mathrm{~min}$ at $30 \%$ power. Different dilutions were then prepared in cell culture medium.

\subsection{Cell viability assay}

Cells were seeded in 96-well plates at a density of $2 \times$ $10^{3}$ per well and treated with reagents for 24,48 , and 72 h. The cytotoxicity of CQD was analyzed with an MTT [3-(4,5-dimethylthiazol-2yl)-2,5-diphenyltetrazolium bromide] assay using Cell Proliferation Kit I according to the manufacturer's instructions. Each experiment was performed in triplicate. Cell viability was expressed as a percentage of the control.

\subsection{Wound healing assay}

Cells were seeded in six-well plates and cultured until confluent. A sterile $200 \mu \mathrm{L}$ pipette tip was used to make a straight scratch, simulating a wound. Cells were then rinsed with PBS (very gently as sheets of cells may detach if care is not exercised) and replaced with $1.5 \mathrm{~mL}$ of medium containing reagents. Cells were photographed using phase contrast and a magnification of $10 \times$, and cells were photographed again 24,48 and $72 \mathrm{~h}$ later. After each measurement, old medium was replaced with fresh medium containing additives.

\subsection{Statistical analysis}

All experiments were performed in triplicate, and results were analyzed via one-way analysis of variance (ANOVA) using GraphPad Prism 4, followed by the Student's $t$-test performed using the Microsoft Office software Excel. $p<0.05$ was considered to indicate a significant difference.

\section{Results and Discussion}

\subsection{Carbon dots inhibited the proliferation of cancer cells}

An MTT assay was used to estimate the extent to which carbon dots inhibited the proliferation of liver cancer cell lines (HepG2, HepG2.2.15, PLC/PRF/5, Huh-7, Huh-1, HLE, HLF, SK-Hep-1, Hep3B, and BEL-7402), pancreatic cancer cell lines (Panc-1, CAPAN-1, MIA-Paca-2, and SUIT-2), an intrahepatic cholangiocarcinoma cell line (RBE), and a colorectal adenocarcinoma cell line (DLD-1). As shown in Figure 2, carbon dots significantly inhibited those cell lines. Cancer cells were treated with various concentrations of carbon dots $(5 \mathrm{mg} / \mathrm{mL}, 0.5 \mathrm{mg} / \mathrm{mL}, 0.05 \mathrm{mg} / \mathrm{mL})$, and proliferation was measured at 24,48 , and $72 \mathrm{~h}$. Results indicated that the inhibitory effect of carbon dots was dose- and time-dependent and that carbon dots most markedly inhibited cell proliferation at a concentration of $5 \mathrm{mg} / \mathrm{mL}$. These findings suggest that carbon dots inhibit the proliferation of liver cancer cells, pancreatic 

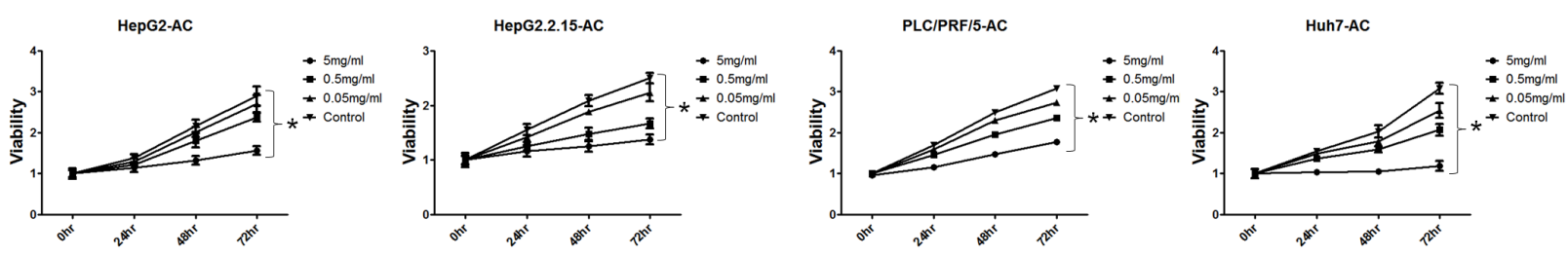

Huh1-AC
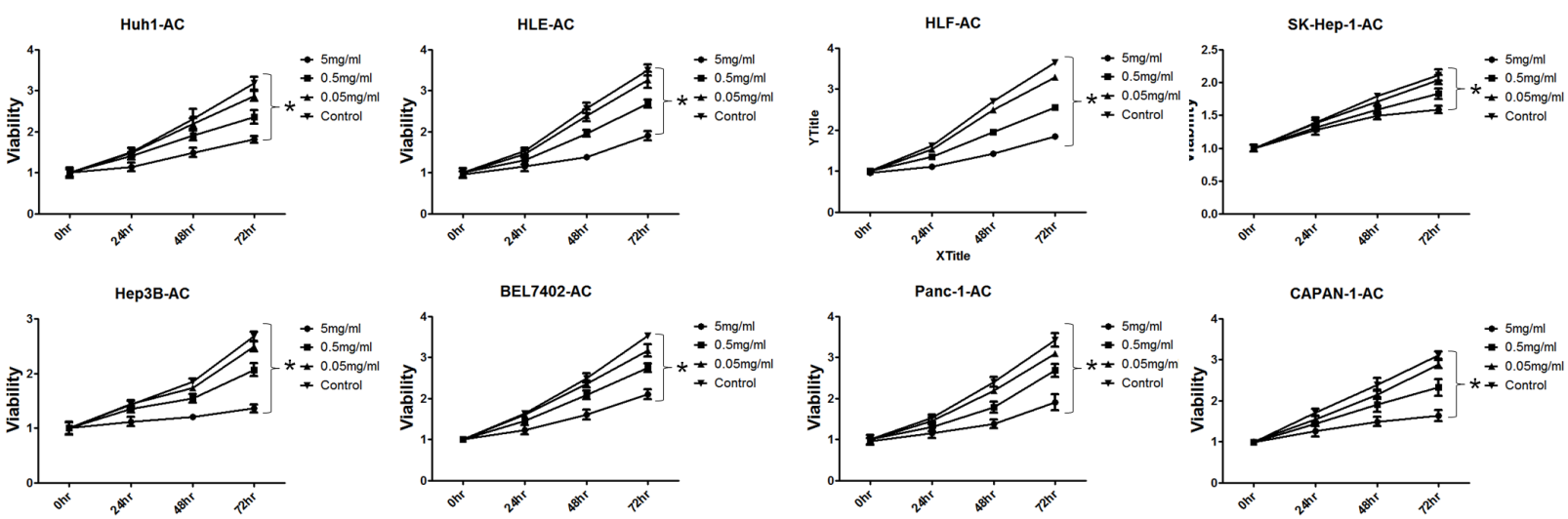

MIA CaPa-2-AC

SUIT-2-AC
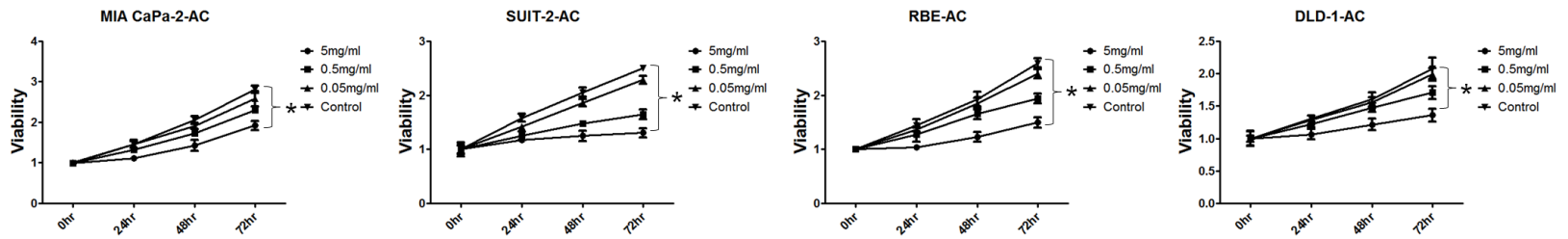

Figure 2. Inhibition of cancer cell growth by carbon dots.

cancer cells, intrahepatic cholangiocarcinoma cells, and colorectal adenocarcinoma cells. They may substantially inhibit other types of cancer cells as well. Further study is needed to determine the molecular mechanism of that inhibitory effect.

\subsection{Carbon dots inhibited the migration of cancer cells}

A wound healing assay was used to investigate the effects of carbon dots on the migratory ability of cancer cells. Six liver cancer cell lines (HLF, SK-Hep-1, Huh-7, HLE, HepG2, and Hep3B) were used in this experiment. After treatment with different reagents for $72 \mathrm{~h}$, marked changes in healing were evident in cancer cells (Figure 3). Changes in the area of the scratch were analyzed in photographs to quantify cell migration. As shown in Figure 3, cells treated with carbon dots had less healing than the control group $(p<0.05)$. These results suggested that carbon dots can decrease the migration of liver cancer cells. Further study is planned to determine if carbon dots act similarly on other types of cancer cells and the molecular mechanism of that action.

\subsection{Combination therapy inhibited cancer cells to a greater extent}

An MTT assay was used to determine the extent to
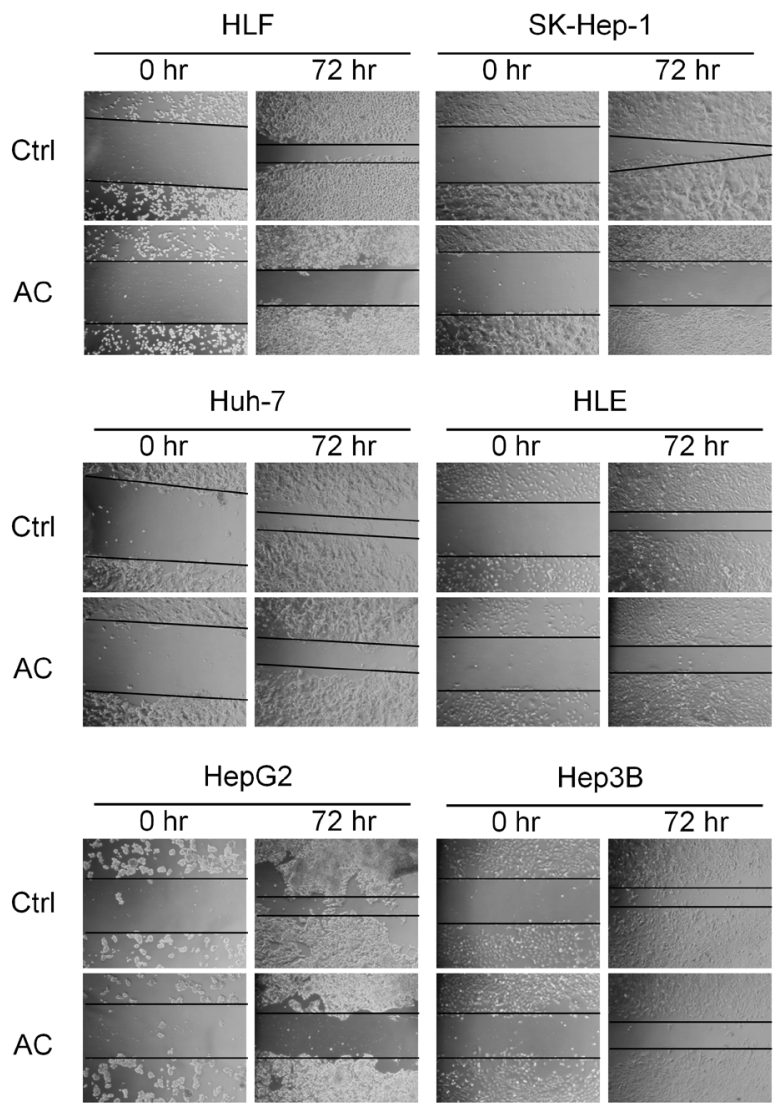

Figure 3. Detection of the extent to which carbon dots inhibited the migration of cancer cells. 

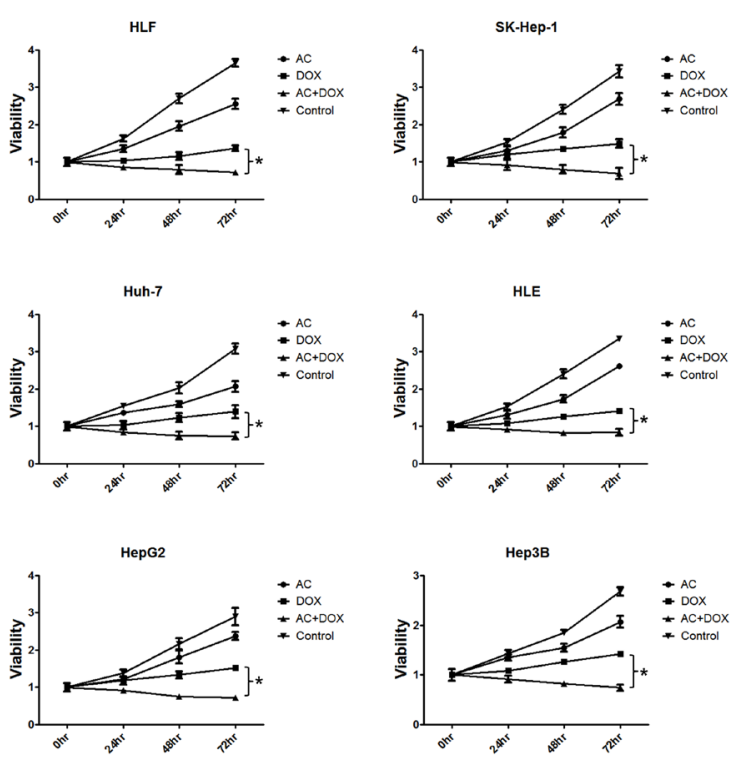

Figure 4. Detection of the extent to which the combined therapies inhibited cancer cells.

which a combination of carbon dots and doxorubicin inhibited cell proliferation. Six liver cancer cell lines (HLF, SK-Hep-1, Huh-7, HLE, HepG2, and Hep3B) were used in this experiment. Four experimental groups were established: a carbon dot group $(0.5 \mathrm{mg} / \mathrm{mL})$, a doxorubicin group $(800 \mathrm{nM})$, a combined therapy group ( $0.5 \mathrm{mg} / \mathrm{mL}$ carbon dots $+800 \mathrm{nM}$ doxorubicin), and a control group. As shown in Figure 4, cell inhibition was greater in the combined therapy group than in either the carbon dot group or the doxorubicin group ( $p<$ 0.05). This may indicate that combined administration could increase the therapeutic efficacy of conventional chemotherapy drugs.

\section{Conclusion}

The current study found that carbon dots prepared from red beans were able to inhibit the proliferation and migration of various types of cancer cells. A combination of carbon dots and a conventional chemotherapy drug inhibited cancer cells more than either therapy alone. Taken together, these findings suggest that carbon dots might be an effective alternative and complementary medicine for the treatment of cancers.

\section{References}

1. World Health Organization. Cancer. 12 September 2018. https://www.who.int/en/news-room/fact-sheets/detail/ cancer (accessed December 19, 2018).

2. International Agency for Research on Cancer. Latest global cancer data: Cancer burden rises to 18.1 million new cases and 9.6 million cancer deaths in 2018. www. iarc.fr (accessed December 5, 2018).
3. Lind MJ. Principles of cytotoxic chemotherapy. Medicine. 2008; 36:19-23.

4. Madan V, Lear JT, Szeimies RM. Non-melanoma skin cancer. Lancet. 2010; 375:673-685.

5. Hill R, Healy B, Holloway L, Kuncic Z, Thwaites $\mathrm{D}$, Baldock C. Advances in kilovoltage x-ray beam dosimetry. Phys Med Biol. 2014; 59:R183-231.

6. Levy MH, Back A, Bazargan S, et al. NCCN clinical practice guidelines in oncology: Palliative care. J Natl Compr Canc Netw. 2009; 4:776-818.

7. Waldmann TA. Immunotherapy: Past, present and future. Nat Med. 2003; 9:269-277.

8. Cassileth BR, Deng G. Complementary and alternative therapies for cancer. Oncologist. 2004; 9:80-89.

9. Beg S, Rizwan M, Sheikh AM, Hasnain MS, Anwer K and Kohli K. Advancement in carbon nanotubes: Basics, biomedical applications and toxicity. J Pharm Pharmacol. 2011; 63:141-163.

10. Lacerda L, Raffa S, Prato M, Bianco A, Kostarelos K. Cell-penetrating CNTs for delivery of therapeutics. Nanotoday. 2007; 2:38-43.

11. Liu Z, Tabakman S, Welsher K, Dai H. Carbon nanotubes in biology and medicine: In vitro and in vivo detection, imaging and drug delivery. Nano Res. 2009; 2:85-120.

12. Arlt M, Haase D, Hampel S, Oswald S, Bachmatiuk A, Klingeler R, Schulze R, Ritschel M, Leonhardt A, Fuessel S, Büchner B, Kraemer K, Wirth MP. Delivery of carboplatin by carbon-based nanocontainers mediates increased cancer cell death. Nanotechnology. 2010; 21:335101.

13. Hampel S, Kunze D, Haase D, Krämer K, Rauschenbach M, Ritschel M, Leonhardt A, Thomas J, Oswald S, Hoffmann V, Büchner B. Carbon nanotubes filled with a chemotherapeutic agent: A nanocarrier mediates inhibition of tumor cell growth. Nanomedicine (Lond). 2008; 3:175-182.

14. Tripisciano C, Krämer K, Taylor A, Borowiak-Palen E. Single-wall carbon nanotubes based anticancer drug delivery system. Chem Phys Lett. 2009; 478:200-205.

15. Erdmann K, Ringel J, Hampel S, Rieger C, Huebner D, Wirth MP, Fuessel S. Chemosensitizing effects of carbon-based nanomaterials in cancer cells: Enhanced apoptosis and inhibition of proliferation as underlying mechanisms. Nanotechnology. 2014; 25:405102.

16. Xie Y, Fan H, Lu W, Yang Q, Nurkesh A, Yeleussizov T, Maipas A, Lu J, Manarbek L, Chen Z, Benassi E. Nuclear MET requires ARF and is inhibited by carbon nanodots through binding to phospho-tyrosine in prostate cancer. Oncogene. 2019; 38:2967-2983.

17. Li CL, Ou CM, Wu WC, et al. Carbon dots prepared from ginger exhibiting efficient inhibition of human hepatocellular carcinoma cells. J Mater Chem B. 2014; 2:4564-4571.

18. Xie Y, Filchakova O, Yang Q, Yesbolatov Y, Tursynkhan D, Kassymbek A, Bouhrara M, Wang K, Balanay M, Fan H. Inhibition of cancer cell proliferation by carbon dots derived from date pits at low dose. Chem Select. 2017; 2:4079-4083.

19. Xie Y, Sun Q, Nurkesh AA, et al. Dysregulation of YAP by ARF stimulated with tea-derived carbon nanodots. Sci Rep. 2017; 7:16577.

(Received March 1, 2019; Revised April 1, 2019; Accepted April 11, 2019) 